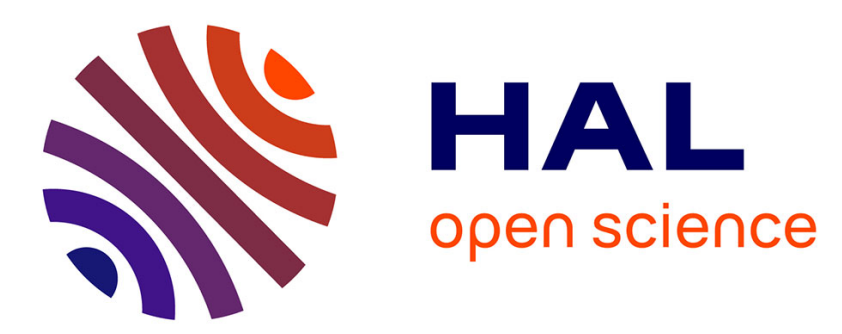

\title{
Selection of Interface DoFs in Hub-blade(s) Coupling of Ampair Wind Turbine Test Bed
}

\author{
Jacopo Brunetti, Antonio Culla, Walter d'Ambrogio, Annalisa Fregolent
}

\section{To cite this version:}

Jacopo Brunetti, Antonio Culla, Walter d'Ambrogio, Annalisa Fregolent. Selection of Interface DoFs in Hub-blade(s) Coupling of Ampair Wind Turbine Test Bed. Mayes, Randy and Rixen, Daniel and Allen, Matt. Topics in Experimental Dynamic Substructuring, 2, Springer New York, pp.167-178, 2014, Conference Proceedings of the Society for Experimental Mechanics Series, 10.1007/978-1-46146540-9_14. hal-00872516

\section{HAL Id: hal-00872516 https://hal.science/hal-00872516}

Submitted on 25 May 2016

HAL is a multi-disciplinary open access archive for the deposit and dissemination of scientific research documents, whether they are published or not. The documents may come from teaching and research institutions in France or abroad, or from public or private research centers.
L'archive ouverte pluridisciplinaire HAL, est destinée au dépôt et à la diffusion de documents scientifiques de niveau recherche, publiés ou non, émanant des établissements d'enseignement et de recherche français ou étrangers, des laboratoires publics ou privés. 


\title{
Selection of Interface DoFs in Hub-Blade(s) Coupling of Ampair Wind Turbine Test Bed
}

\author{
Jacopo Brunetti*, Antonio Culla**, Walter D’Ambrogio*†, Annalisa Fregolent ${ }^{* *}$ \\ * Dipartimento di Ingegneria Industriale e dell'Informazione e di Economia, Università dell'Aquila \\ Via G. Gronchi, 18 - I-67100, L’Aquila (AQ), Italy, ${ }^{\dagger}$ email: walter . dambrogio@univaq.it \\ ** Dipartimento di Ingegneria Meccanica e Aerospaziale, Università di Roma La Sapienza \\ Via Eudossiana 18, I 00184 Rome, Italy
}

\begin{abstract}
Substructure coupling is an important tool in several applications of modal analysis. It is particularly relevant in virtual prototyping of complex systems and responds to actual industrial needs, especially in an experimental context. Furthermore, the reverse problem, the decoupling of a substructure from an assembled system, arises when a substructure cannot be tested separately but only when coupled to neighboring substructures, a situation often encountered in practice. In this paper, the dynamic behavior of the Ampair test bed wind turbine rotor, made by three blades - each one bolted to the hub at three points - is analyzed. The aim is both to identify the dynamic behavior of the rotor starting from the frequency response functions (FRFs) of blades and hub, and to select a reduced set of relevant DoFs to represent the interface between blades and hub. FRFs to be used in the coupling procedure are obtained starting from FE model of each substructure, by using a super-element based computational approach. The decoupling problem, with the aim of identifying the dynamic behavior of each blade from the FRFs of the assembled rotor and of the hub, is also considered.
\end{abstract}

Keywords: Experimental Dynamic Substructuring, Interface DoFs, Wind Turbine

\section{INTRODUCTION}

In experimental dynamic substructuring two main problems can be defined: addition of substructures (coupling) and subtraction of substructures (decoupling). Coupling is important to find out the dynamic behaviour of complex structures from a dynamic description of their components. It is particularly relevant in virtual prototyping of complex systems and responds to actual industrial needs, especially in an experimental context, i.e. when the model of at least one subsystem derives from experimental tests. Decoupling can be important in built-up structures where some components (critical subsystems or joints) cannot be removed or accessed easily. Decoupling involves the identification of the dynamic behaviour of a structural subsystem, starting from information about the remaining part of the structural system (residual subsystem) and from the known dynamic behaviour of the complete system.

Addition of substructures (coupling) can be seen as a structural modification problem [1]. Similarly, the decoupling problem can be seen as a structural modification problem with negative modification. Due to modal truncation problems, in experimental dynamic substructuring, the use of FRFs (Frequency Based Substructuring) is preferred with respect to the use of modal parameters. The main algorithm for frequency based substructuring is the improved 
impedance coupling [2] that involves just one matrix inversion with respect to the classical impedance coupling technique that requires three inversions. A general framework for dynamic substructuring is provided in $[3,4]$ : in this context, the so called dual domain decomposition is very useful for experimental application, since it allows to retain the full set of global DoFs by ensuring equilibrium at the interface between substructures. A similar formulation for the decoupling problem is developed and discussed in [5-8].

Whatever be the used approach, the dynamic behaviour (FRFs) at all the coupling DoFs must be determined. Therefore, if coupling involves transmission of moments, either rotational measurements or techniques involving a suitable selection of translational DoFs are required, such as the equivalent multi point connection $[4,9]$ or the transmission simulator method $[10,11]$. With regard to the decoupling problem, additional FRFs at some internal DoFs of the residual subsystem can be used $[6,12,13]$ to avoid ill-conditioning at some particular frequencies.

In this paper, dynamic substructuring is applied to the rotor of the Ampair 600 wind turbine. This system has been proposed as a test bed by the Society of Experimental Mechanics focus group on experimental dynamic susbstructuring, to enable advancements in experimental dynamic substructuring technology and theory. Several specimens of the turbine have been bought. A description of the turbine with modifications made to the system to make it more linear is presented in [14] together with results from a rudimentary modal test on the whole turbine. The results of initial modal tests on six blades are reported in [15]. Geometry data about the blades and the hub have also been made available to members of the focus group.

The aim of this paper is to select a reduced set of relevant DoFs to describe the interface between each blade and the hub in order to identify the dynamic behavior of the rotor starting from the FRFs of the blades and the hub. FRFs to be used in the coupling procedure are obtained starting from the FE model of each substructure, by using a super-element based computational approach. The choice of using computational FRFs is dictated by the need of being aware of the effect of each simplifying assumption, by comparing the results obtained at each step with the reference ones. The decoupling problem, with the aim of identifying the dynamic behavior of each blade from the FRFs of the assembled rotor and of the hub, is also considered

\section{THEORETICAL BACKGROUND}

The coupled structural system is assumed to be made by two (or more) subsystems joined through a number of couplings (see Fig. 1). The degrees of freedom (DoFs) of the coupled system can be partitioned into internal DoFs (not belonging to the couplings) and coupling DoFs (c).

\subsection{Addition of subsystems}

If addition of subsystems (coupling problem) is considered, all subsystems are assumed to be known whilst the FRF of the coupled system is unknown. In the frequency domain, the equation of motion of a linear time-invariant subsystem $r$ may be written as:

$$
\mathbf{Z}^{(\mathbf{r})}(\omega) \mathbf{u}^{(\mathbf{r})}(\omega)=\mathbf{f}^{(\mathbf{r})}(\omega)+\mathbf{g}^{(\mathbf{r})}(\omega)
$$

where:

$\mathbf{Z}^{(r)}$ is the dynamic stiffness matrix of subsystem $r$;

$\mathbf{u}^{(r)}$ is the vector of degrees of freedom of subsystem $r$;

$f^{(r)}$ is the external force vector;

$\mathbf{g}^{(\mathbf{r})}$ is the vector of connecting forces with other subsystems (constraint forces arising from compatibility conditions). 


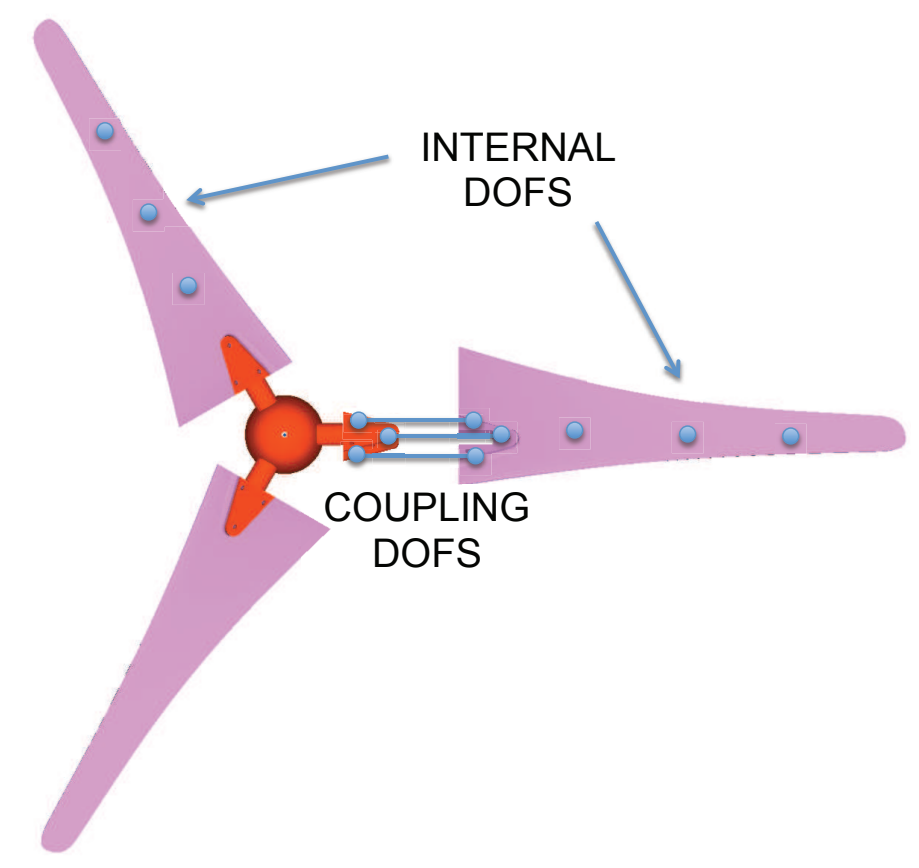

Figure 1: Scheme of the substructuring problem.

For the sake of simplicity, the explicit frequency dependence will be omitted. Furthermore, the procedure will be developed with reference to two subsystems, bearing in mind that it can be easily extended to more subsystems.

The equation of motion of the subsystems to be coupled can be written in a block diagonal format as:

$$
\mathbf{Z} \mathbf{u}=\mathbf{f}+\mathbf{g} \quad \text { i.e. } \quad\left[\begin{array}{cc}
\mathbf{Z}^{(1)} & 0 \\
0 & \mathbf{Z}^{(2)}
\end{array}\right]\left\{\begin{array}{l}
\mathbf{u}^{(1)} \\
\mathbf{u}^{(2)}
\end{array}\right\}=\left\{\begin{array}{l}
\mathbf{f}^{(1)} \\
\mathbf{f}^{(2)}
\end{array}\right\}+\left\{\begin{array}{l}
\mathbf{g}^{(1)} \\
\mathbf{g}^{(2)}
\end{array}\right\}
$$

The compatibility condition at the interface DoFs implies that any pair of matching DoFs $u_{1}^{(1)}$ and $u_{m}^{(2)}$, i.e. DoF / on subsystem 1 and DoF $m$ on subsystem 2 must have the same displacement, that is $u_{1}^{(1)}-u_{m}^{(2)}=0$.

This condition can be generally expressed as:

$$
\mathbf{B u}=0 \quad \text { i.e. } \quad\left[\begin{array}{ll}
\mathbf{B}^{(1)} & \mathbf{B}^{(2)}
\end{array}\right]\left\{\begin{array}{l}
\mathbf{u}^{(1)} \\
\mathbf{u}^{(2)}
\end{array}\right\}=0
$$

where each row of $\mathbf{B}$ corresponds to a pair of matching DoFs. Note that $\mathbf{B}$ is, in most cases, a signed Boolean matrix and it can be written by distinguishing the contribution of the different subsystems.

The equilibrium condition for constraint forces associated with the compatibility conditions implies that, when the connecting forces are added for a pair of matching DoFs, their sum must be zero, i.e. $g_{l}^{(1)}+g_{m}^{(2)}=0$ : this holds for any pair of matching DoFs. Furthermore, if DoF $k$ on subsystem 1 (or 2) is not a connecting DoF, it must be $g_{k}^{(1)}=0$ : this holds for any non-interface DoF. 
Overall, the above conditions can be expressed as:

$$
\mathbf{L}^{T} \mathbf{g}=0
$$

where the matrix $\mathbf{L}$ is a Boolean localisation matrix. Note that the number of rows of $\mathbf{L}^{T}$ is equal to the number of non-interface DoFs plus the number of pairs of interface DoFs.

Eqs. (2-4) can be put together to obtain the so called 3-field formulation [3]:

$$
\left\{\begin{array}{l}
Z \mathbf{u}=\mathbf{f}+\mathbf{g} \\
\mathbf{B u}=0 \\
\mathbf{L}^{T} \mathbf{g}=0
\end{array}\right.
$$

\subsubsection{Dual formulation in the frequency domain [3]}

In the dual formulation, the total set of DoFs is retained, i.e. each interface DoF is present as many times as there are substructures connected through that DoF. The equilibrium condition $g_{l}^{(1)}+g_{m}^{(2)}=0$ at a pair of interface DoFs is ensured by choosing, for instance, $g_{l}^{(1)}=-\lambda$ and $g_{m}^{(2)}=\lambda$. Due to the construction of $\mathbf{B}$, the overall interface equilibrium can be ensured by writing the connecting forces in the form:

$$
\mathbf{g}=-\mathbf{B}^{T} \lambda
$$

where $\lambda$ are Lagrange multipliers corresponding to connecting force intensities. Since there is a unique set of connecting force intensities $\lambda$, the interface equilibrium condition (4) is satisfied automatically for any $\lambda$, i.e.

$$
\mathbf{L}^{T} \mathbf{g}=-\mathbf{L}^{T} \mathbf{B}^{T} \lambda=0
$$

Then $\mathbf{B}^{T}$ is the nullspace of $\mathbf{L}^{T}$, so Eq. (7) is always satisfied and the system of equations (5) becomes:

$$
\left\{\begin{array}{l}
\mathbf{Z u}+\mathbf{B}^{T} \lambda=\mathbf{f} \\
\mathbf{B u}=0
\end{array}\right.
$$

In matrix notation:

$$
\left[\begin{array}{cc}
\mathbf{Z} & \mathbf{B}^{T} \\
\mathbf{B} & 0
\end{array}\right]\left\{\begin{array}{l}
\mathbf{u} \\
\lambda
\end{array}\right\}=\left\{\begin{array}{l}
\mathbf{f} \\
0
\end{array}\right\}
$$

that is: 


$$
\left[\begin{array}{ccc}
\mathbf{Z}^{(1)} & 0 & \mathbf{B}^{(1)} \\
0 & \mathbf{Z}^{(2)} & \mathbf{B}^{(2)} \\
\mathbf{B}^{(1)} & \mathbf{B}^{(2)} & 0
\end{array}\right]\left\{\begin{array}{c}
\mathbf{u}^{(1)} \\
\mathbf{u}^{(2)} \\
\lambda
\end{array}\right\}=\left\{\begin{array}{c}
\mathbf{f}^{(1)} \\
\mathbf{f}^{(2)} \\
0
\end{array}\right\}
$$

Note that $\mathbf{B}^{(1)}$ and $\mathbf{B}^{(2)}$ extract the coupling DoFs among the full set of DoFs.

By eliminating $\lambda$, it is possible to obtain a relation in the form $\mathbf{u}=\mathbf{H f}$, which provides the FRF of the coupled system [6]:

$$
\mathbf{u}=\left(\mathbf{Z}^{-1}-\mathbf{Z}^{-1} \mathbf{B}^{T}\left(B \mathbf{Z}^{-1} \mathbf{B}^{T}\right)^{-1} \mathbf{B} \mathbf{Z}^{-1}\right) \mathbf{f}
$$

In expanded notation:

$$
\begin{aligned}
\left\{\begin{array}{l}
\mathbf{u}^{(1)} \\
\mathbf{u}^{(2)}
\end{array}\right\}= & \left(\left[\begin{array}{cc}
\mathbf{Z}^{(1)} & 0 \\
0 & \mathbf{Z}^{(2)}
\end{array}\right]^{-1}-\left[\begin{array}{cc}
\mathbf{Z}^{(1)} & 0 \\
0 & \mathbf{Z}^{(2)}
\end{array}\right]^{-1}\left[\begin{array}{l}
\mathbf{B}^{(1)} \\
\mathbf{B}^{(2)}
\end{array}\right] \times\right. \\
& \left.\times\left(\left[\begin{array}{ll}
\mathbf{B}^{(1)} & \mathbf{B}^{(2)}
\end{array}\right]\left[\begin{array}{cc}
\mathbf{Z}^{(1)} & 0 \\
0 & \mathbf{Z}^{(2)}
\end{array}\right]^{-1}\left[\begin{array}{l}
\mathbf{B}^{(1)^{T}} \\
\mathbf{B}^{(2)}
\end{array}\right]\right)^{-1}\left[\begin{array}{ll}
\mathbf{B}^{(1)} & \mathbf{B}^{(2)}
\end{array}\right]\left[\begin{array}{cc}
\mathbf{Z}^{(1)} & 0 \\
0 & \mathbf{Z}^{(2)}
\end{array}\right]^{-1}\right)\left\{\begin{array}{l}
\mathbf{f}^{(1)} \\
\mathbf{f}^{(2)}
\end{array}\right\}
\end{aligned}
$$

i.e., by introducing the FRFs $\mathbf{H}^{(1)}$ and $\mathbf{H}^{(2)}$ at the full set of DoFs instead of $\left[\mathbf{Z}^{(1)}\right]^{-1}$ and $\left[\mathbf{Z}^{(2)}\right]^{-1}$ :

$$
\mathbf{H}^{\mathbf{R U}}=\left[\begin{array}{cc}
\mathbf{H}^{(1)} & 0 \\
0 & \mathbf{H}^{(2)}
\end{array}\right]-\left[\begin{array}{cc}
\mathbf{H}^{(1)} & 0 \\
0 & \mathbf{H}^{(2)}
\end{array}\right]\left[\begin{array}{l}
\mathbf{B}^{(1)^{T}} \\
\mathbf{B}^{(2)^{T}}
\end{array}\right]\left(\left[\begin{array}{ll}
\mathbf{B}^{(1)} & \mathbf{B}^{(2)}
\end{array}\right]\left[\begin{array}{cc}
\mathbf{H}^{(1)} & 0 \\
0 & \mathbf{H}^{(2)}
\end{array}\right]\left[\begin{array}{l}
\mathbf{B}^{(1)^{T}} \\
\mathbf{B}^{(2)^{T}}
\end{array}\right]\right)^{-1}\left[\begin{array}{ll}
\mathbf{B}^{(1)} & \mathbf{B}^{(2)}
\end{array}\right]\left[\begin{array}{cc}
\mathbf{H}^{(1)} & 0 \\
0 & \mathbf{H}^{(2)}
\end{array}\right]
$$

With the dual formulation, the rows and columns corresponding to the coupling DoFs appear twice in $\mathbf{H}^{\mathrm{RU}}$. Obviously, only independent entries are retained.

\subsection{Subtraction of subsystems using the dual domain decomposition [6]}

If subtraction of subsystems (decoupling problem) is considered, the coupled structural system $R U$ and a residual subsystem $R$ are assumed to be known whilst the FRF of subsystem $U$ is unknown. The unknown subsystem $(U)$ and the residual subsystem $(R)$ joined through a number of couplings (see Fig. 2). The degrees of freedom (DoFs) of the coupled system can be partitioned into internal DoFs (not belonging to the couplings) of subsystem $U(u)$, internal DoFs of subsystem $R(r)$, and coupling DoFs $(c)$.

It is required to find the FRF of the unknown substructure $U$ starting from the FRF of the coupled system $R U$. The subsystem $U$ can be extracted from the coupled system $R U$ by cancelling the dynamic effect of the residual subsystem $R$. This can be accomplished by adding to the coupled system $R U$ a fictitious subsystem with a dynamic stiffness opposite to that of the residual subsystem $R$ and satisfying compatibility and equilibrium conditions. 


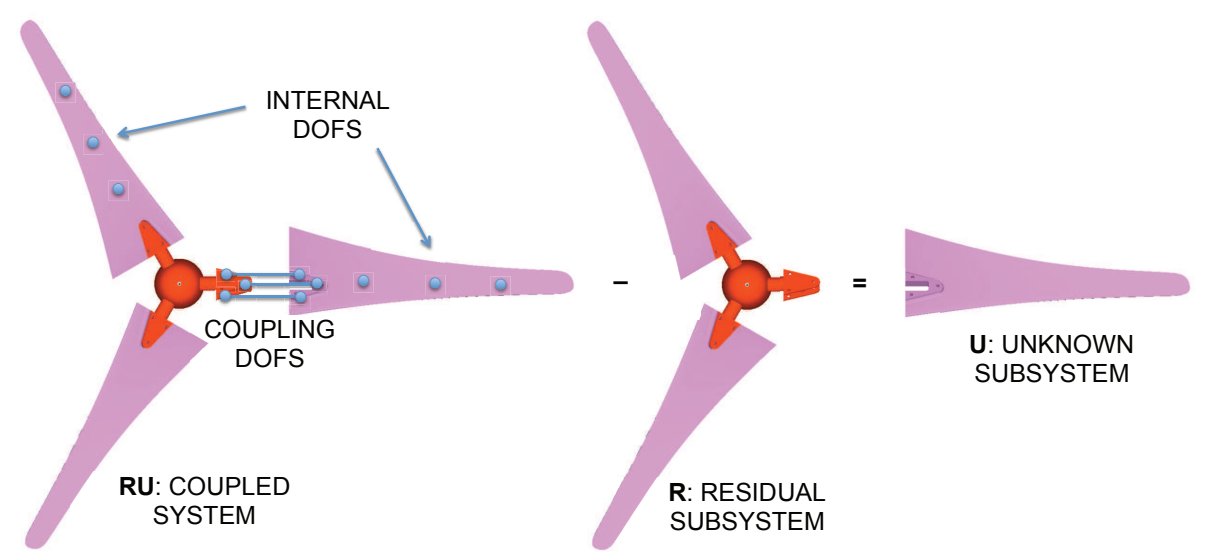

Figure 2: Scheme of the decoupling problem.

According to this point of view, the interface between the coupled system $R U$ and the fictitious subsystem should not only include the coupling DoFs between subsystems $U$ and $R$, but should as well include the internal DoFs of subsystem $R$. However, it can be shown that the problem can be solved by considering a number of interface DoFs at least equal to the number of coupling DoFs $n_{c}$. Therefore, three options for interface DoFs can be considered:

- standard interface, including only the coupling DoFs $(c)$ between subsystems $U$ and $R$;

- extended interface, including also some internal DoFs $(i \subseteq r)$ of the residual substructure;

- mixed interface, including some coupling DoFs $(d \subseteq c)$ and some internal DoFs $(i \subseteq r)$ of the residual substructure.

In the framework of the dual formulation in the frequency domain (see Section 2.1.1), the union between the coupled system $R U$ and the fictitious subsystem can be written (see Eq. 10) as:

$$
\left[\begin{array}{ccc}
\mathbf{Z}^{\mathrm{RU}} & 0 & \mathbf{B}^{\mathrm{RU}} \\
0 & -\mathbf{Z}^{\mathrm{R}} & \mathbf{B}^{\mathbf{R}^{T}} \\
\mathbf{B}^{\mathrm{RU}} & \mathbf{B}^{\mathrm{R}} & 0
\end{array}\right]\left\{\begin{array}{c}
\mathbf{u}^{\mathrm{RU}} \\
\mathbf{u}^{\mathrm{R}} \\
\lambda
\end{array}\right\}=\left\{\begin{array}{c}
\mathbf{f}^{\mathrm{RU}} \\
\mathbf{f}^{\mathrm{R}} \\
0
\end{array}\right\}
$$

Following the same procedure used in Section 2.1.1, it is possible to obtain the FRF of the unknown subsystem $U$.

$$
\mathbf{H}^{\mathbf{U}}=\left[\begin{array}{cc}
\mathbf{H}^{\mathrm{RU}} & 0 \\
0 & -\mathbf{H}^{\mathbf{R}}
\end{array}\right]-\left[\begin{array}{cc}
\mathbf{H}^{\mathrm{RU}} & 0 \\
0 & -\mathbf{H}^{\mathbf{R}}
\end{array}\right]\left[\begin{array}{c}
\mathbf{B}^{\mathrm{RU}}{ }^{T} \\
\mathbf{B}^{\mathbf{R}^{T}}
\end{array}\right]\left(\left[\begin{array}{ll}
\mathbf{B}^{\mathbf{R U}} & \mathbf{B}^{\mathbf{R}}
\end{array}\right]\left[\begin{array}{cc}
\mathbf{H}^{\mathbf{R U}} & 0 \\
0 & -\mathbf{H}^{\mathbf{R}}
\end{array}\right]\left[\begin{array}{c}
\mathbf{B}^{\mathbf{R U}} \\
\mathbf{B}^{\mathbf{R}^{T}}
\end{array}\right]\right)^{-1}\left[\begin{array}{cc}
\mathbf{B}^{\mathbf{R U}} & \mathbf{B}^{\mathbf{R}}
\end{array}\right]\left[\begin{array}{cc}
\mathbf{H}^{\mathrm{RU}} & 0 \\
0 & -\mathbf{H}^{\mathbf{R}}
\end{array}\right]
$$

Note that $\mathbf{H}^{\mathbf{R U}}$ and $\mathbf{H}^{\mathbf{R}}$ are the FRFs at the full set of DoFs of the coupled system and the residual subsystem. 

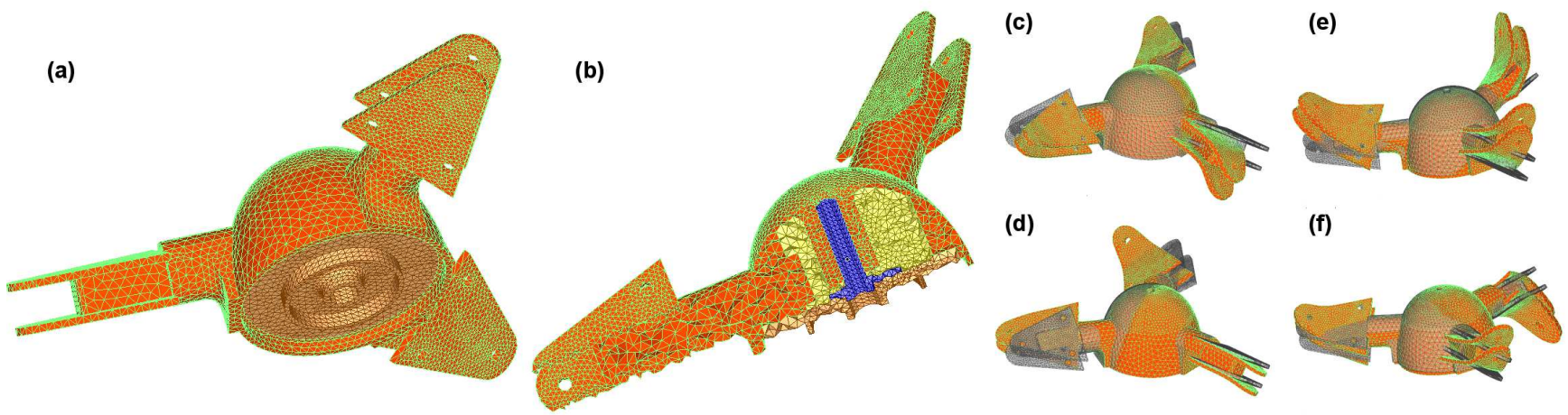

Figure 3: Assembled Hub FE model (a), a cross section of the model showing the different parts of the assembly (b) and the first four modal shapes (c,d,e,f).
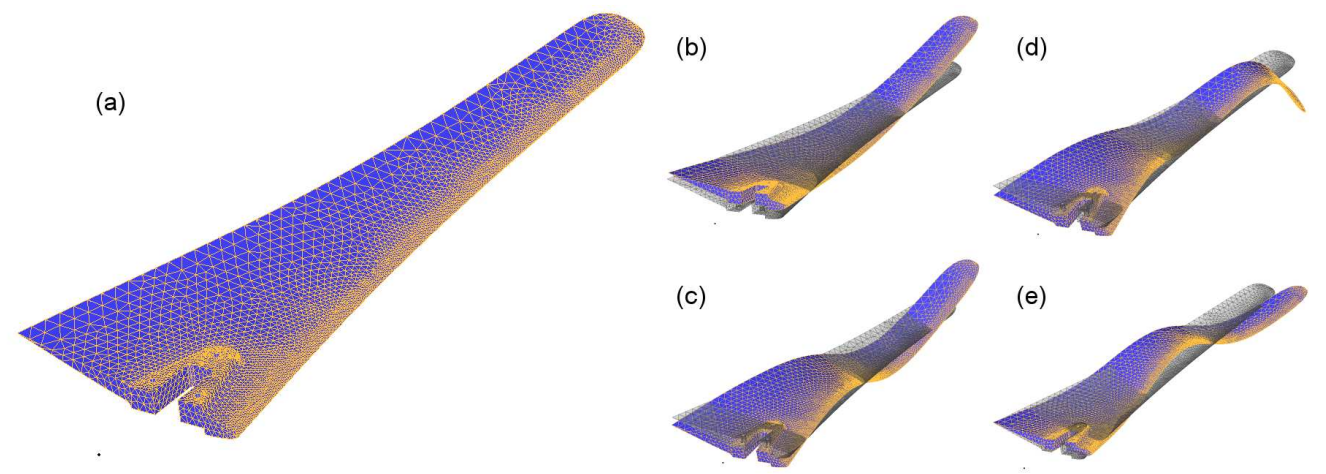

Figure 4: FEM model of the blade (a) and first four modal shapes (b,c,d,e).

\section{CASE STUDY}

In this paper the dynamic behavior of the rotor of the Ampair 600 wind turbine is analyzed. The rotor is made by three blades clamped to the hub by three bolts and two plates that sandwich the blade. The blades are made of glass reinforced polyester and they are coated with a white epoxy. The hub to which the blades are attached has an overspeed mechanism to vary the pitch of the blades: the mechanism is activated by centrifugal masses that are fixed through bolts in the holes at the greatest radial distance from the rotation axis of the hub.

One of the most interesting features in the study of coupling and decoupling problem is the possibility to use both experimental and numerical data. In this case a Finite Element Model of the whole rotor is developed to extract the Frequency Response Functions needed to apply the Frequency Based Substructuring methods. In this model all the alteration to the commercial turbine introduced to reduce the nonlinear behavior are considered. The overspeed mechanism was potted to reduce the rattling and the free play between the different parts of the mechanism and it is not considered in this modeling. Moreover, the centrifugal masses that activate the mechanism are not taken into account and lastly the internal shaft is considered to be directly bonded to the hub.

Four substructures are defined inside the whole rotor: the central hub and the three blades which are identical to each other, so only two different substructures have to be modeled to find the dynamic behavior of the whole system. The hub is made of isotropic material whit a negligible damping. For the sake of simplicity it is modeled entirely in Aluminum Alloy, keeping the chance to eventually consider different materials for each component. The Finite Element Model of the hub (Fig. 3) is made of around 130000 tetrahedral elements with linear shape function. The blades are made of Fiber Reinforced Polyester but the mechanical properties are unknown. Here a linear material is considered and the Finite Element Model of each blade (Fig. 4) is made of around 50000 tetrahedral elements. 


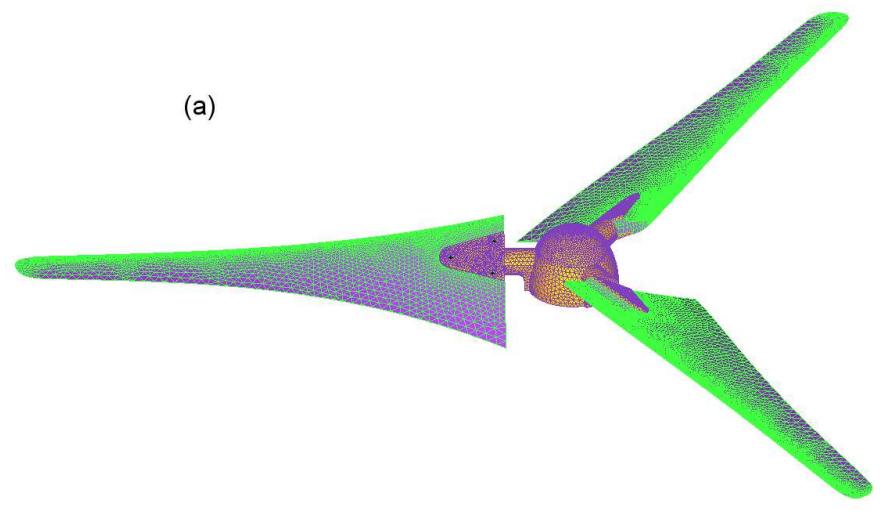

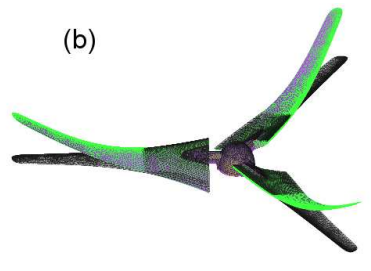

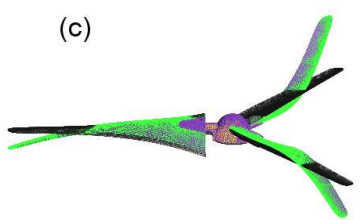

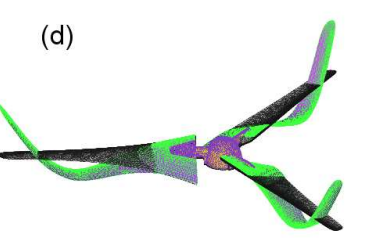

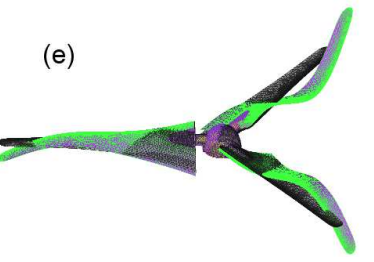

Figure 5: FEM model of the rotor (a) and first four modal shapes (b,c,d,e).

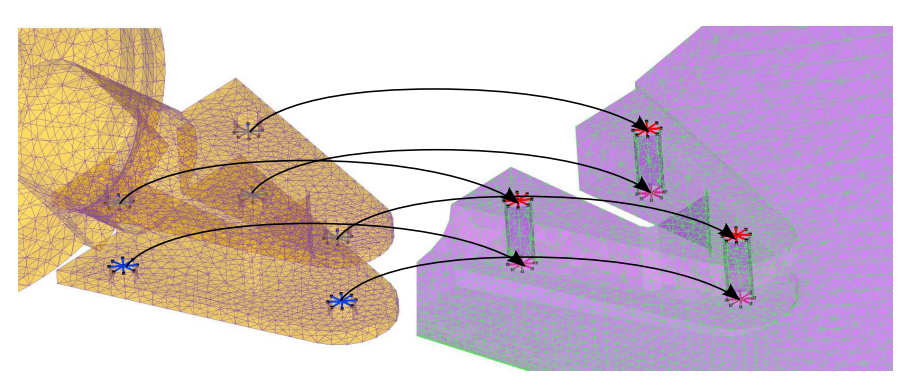

Figure 6: RBE at the interface.

The whole rotor, composed by the three blades fixed on the central hub, is also modeled (Fig. 5) with the aim to compare its behavior to the coupling results and to approach the decoupling of the system. Although the connection between the substructures is ensured by contacting surfaces, efforts to simplify as much as possible the interface are undertaken, with the aim to minimize the DoFs involved in the coupling without losing important information about the dynamic behavior of the interface. After several analysis the connection between the substructures is modeled by enforcing compatibility only at the translational DoFs of the master nodes of the Rigid Body Elements (RBEs) which are realized on both edges of each hole (Fig. 6). This 6 points connection (18 DoF) introduces a relative error lower than $3 \%$ in the determination of the first modal frequencies in the range $0-250 \mathrm{~Hz}$ compared to the modal frequencies when the compatibility is enforced on each node on the contact surface. Moreover this highlights the low influence of the rotational DoFs in the connecting points considered.

To have an idea of the dynamic behavior of the system and of the subsystems, the natural frequencies of the blade subsystems, of the hub subsystem and of the coupled rotor system are shown in Table 1. For the coupled system, the values are referred to the simplified connection described above.

Receptance matrices to be used for the coupling with Frequency Based Substructuring techniques are obtained through a superelement based computational approach (Fig. 7). In this way, the receptance matrix is determined only on a subset of physical points that are relevant to solve the coupling problem. First, superelements are defined using a Craig-Bampton reduction of the Finite Element Model; then, the receptance matrix is directly computed using the mass and stiffness matrices of the reduced system. In the Craig-Bampton method, the reduced mass $\mathbf{M}$ and stiffness $\mathbf{K}$ matrices are defined for a subset of physical DoFs and a set of fictitious DoFs representing the modes of the structure constrained at the connecting points. After condensation, the reduced mass and stiffness matrices are extracted from the FEM and imported in Matlab environment to find the receptance matrix of the subsystem at each frequency of interest $\mathbf{H}(\omega)=\left[\mathbf{K}-\omega^{2} \mathbf{M}\right]^{-1}$. 
TABLE 1: Natural frequencies of the systems [Hz]

\begin{tabular}{llll}
\hline & \multicolumn{3}{c}{ System } \\
\cline { 2 - 4 } Mode & Blade & Hub & Rotor \\
\hline $1-6$ & 0 & 0 & 0 \\
7 & 42,64 & 1977,77 & 21,43 \\
8 & 123,93 & 1977,77 & 28,77 \\
9 & 238,6 & 2284,54 & 28,77 \\
10 & 251,83 & 3091,28 & 73,34 \\
11 & 329,22 & 3091,29 & 76,62 \\
12 & 430,24 & 3424,74 & 76,63 \\
13 & 532,22 & 3643,71 & 124,47 \\
14 & 702,64 & 3643,79 & 124,47 \\
15 & 781,74 & 3643,81 & 165,08 \\
16 & 942,22 & 4863,77 & 175,97 \\
\hline
\end{tabular}

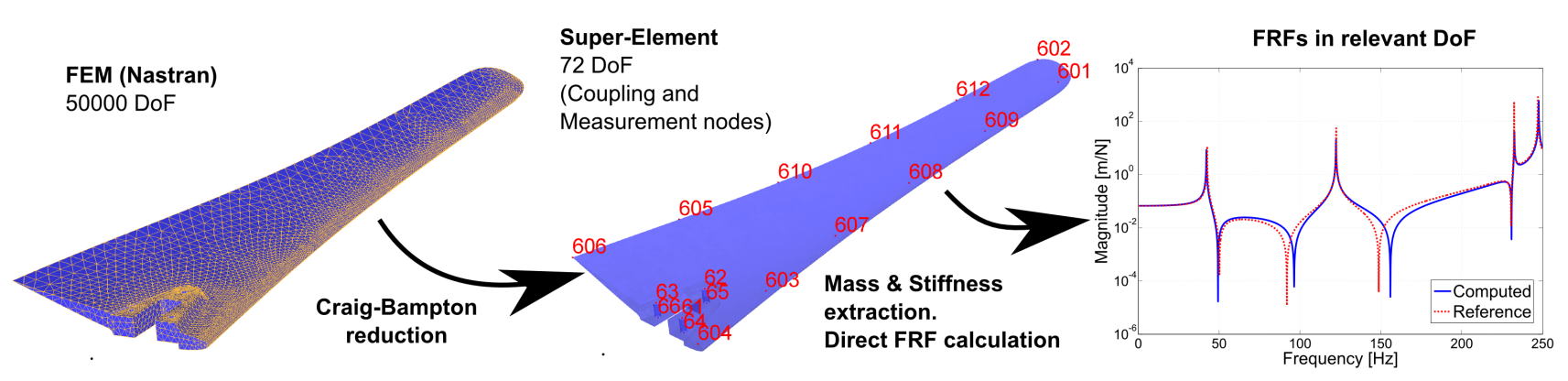

Figure 7: SuperElement computational approach for the determination of the receptance matrix.

\subsection{Coupling one blade with the rest of the rotor}

Coupling between substructures is performed using the dual formulation. The coupled receptance matrix $\mathbf{H}_{\mathbf{C}}$ is defined as:

$$
\mathbf{H}_{\mathbf{C}}=\mathbf{H}-\mathbf{H B}^{T}\left(\mathbf{B H B}^{T}\right)^{-1} \mathbf{B H}
$$

where $\mathbf{H}$ is the receptance matrix of the whole system assembled using the dual approach, and $\mathbf{B}$ is the signed boolean matrix that defines the compatibility condition.

The receptance matrix of the hub substructure $\left(\mathrm{H}^{\mathrm{H}}\right)$ is determined at 18 coupling nodes $(6$ for each blade, see Fig. 8a), by considering 16 modes for the Craig Bampton reduction. The receptance matrix of the blade substructure $\left(\mathbf{H}^{\mathbf{B}}\right)$ is determined at 18 nodes (6 coupling nodes and 12 internal nodes, see Fig. 8b), by considering also in this case 16 modes for the $\mathrm{CB}$ reduction. To analyze the single coupling interface, a substructure composed by the hub and 2 blades is also considered, in this case the receptance matrix $\left(\mathbf{H}^{\mathrm{H}+2 \mathbf{B}}\right)$ is defined at 42 nodes (6 coupling nodes and 36 internal nodes, see Fig. 8c). In this case, due to the more complex behavior of the substructures, 20 modes are considered for the CB reduction.

The reference system of the blade is different from the reference system of the hub, so a rotation is applied to the receptance matrix of the blade, in order that the two reference systems are coincident and the matrix $\mathbf{B}$ is boolean. 


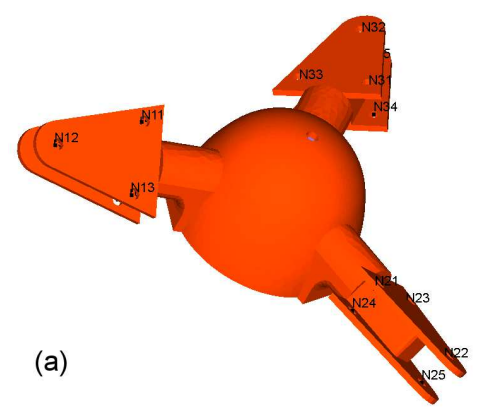

(b)
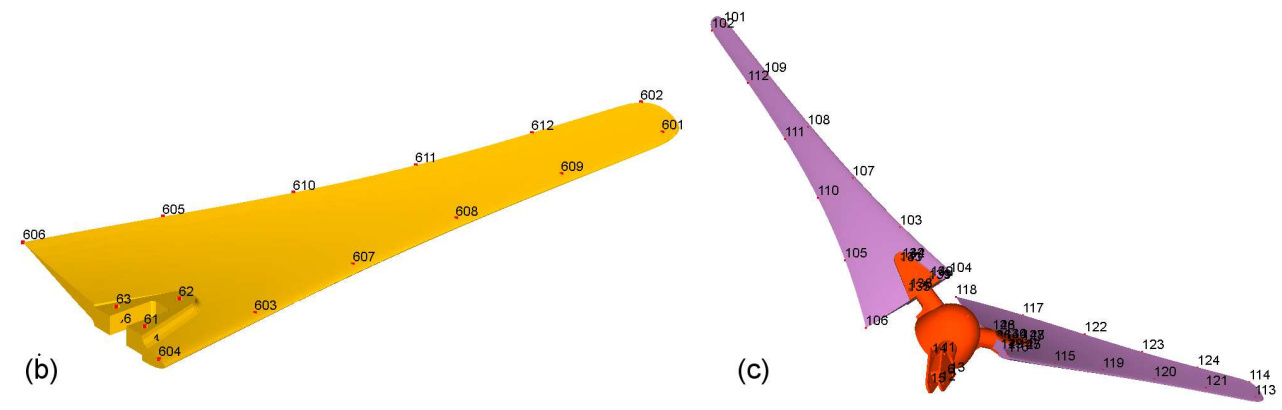

Figure 8: Relevant nodes for coupling in the hub (a), in each blade (b) and in the subsystem composed by the hub and 2 blades (c).

The two substructures (hub with two blades + blade) are coupled using the dual approach. The receptance matrices of the substructures have size $\mathbf{H}^{\mathrm{H}+2 \mathrm{~B}}=[126 \times 126]$ and $\mathbf{H}^{\mathrm{B}}=[54 \times 54]$. The interface among the two substructures is first assumed at the 18 translational DoF of the 6 coupling nodes. The receptance matrix assembled in the dual way is expressed as:

$$
\mathbf{H}=\left[\begin{array}{cc}
\mathbf{H}_{[126 \times 126]}^{\mathbf{H}+2 \mathbf{B}} & \mathbf{O}_{[126 \times 54]} \\
\mathbf{O}_{[54 \times 126]} & \mathbf{H}_{[54 \times 54]}^{\mathbf{B}}
\end{array}\right]
$$

The matrix $\mathbf{B}$ that defines the compatibility condition is:

$$
\mathbf{B}=\left[\begin{array}{llll}
\mathbf{I}_{[18 \times 18]} & 0_{[18 \times 114]} & -\mathbf{I}_{[18 \times 18]} & 0_{[18 \times 36]}
\end{array}\right]
$$

where matrices $\mathbf{I}_{[\mathbf{n} \times \mathbf{n}]}$ and $\mathbf{O}_{[\mathbf{n} \times \mathbf{m}]}$ are respectively the square diagonal unit matrix of size $n$ and the null matrix of size $[n \times m]$.

The receptance matrix of the assembled system is computed using Eq. (17) with a frequency resolution of $0.1 \mathrm{~Hz}$. The natural frequencies (estimated from peaks of the receptance modulus) are determined very accurately: in fact, the coupling procedure itself does not introduce any error in the natural frequencies, when compared to the natural frequencies obtained from the FEM with the simplified interface shown in Fig. 6.

However, a large number of measurements should be performed to determine the receptance matrices of the substructures at all the translational DoFs of the coupling nodes. Therefore, efforts are undertaken to reduce the number of DoFs involved in the coupling procedure. The aim is to find a subset of relevant DoFs to solve the coupling problem without losing information.

After several analyses, a subset of 9 relevant DoFs is found (see Fig. 9a). In order to have a limited error, the selected DoFs should be able to represent the type of connection that in this case is a rigid joint, enforcing constraints on 6 DoFs. So all the translational and rotational actions should be reproduced through forces acting on the selected DoFs.

Trying to further reduce the number of interface DoFs, using for instance the set of 7 DoFs shown in Fig. 9b, drastically worsens the results. The Frequency Response Function for the selected subset of 9 DoFs and for the further reduced subset composed of 7 DoFs is shown in Fig. 10 compared to the reference model. The subset composed by 7 DoFs introduces a much larger error compared to the subset of 9 DoFs. Analyzing the FRFs and reading the values of the natural frequencies in Table 2, the whole FRF appears to be shifted to the left: this is an effect of a drop in the stiffness of the joint, due to the removal of some coupling DoFs. 


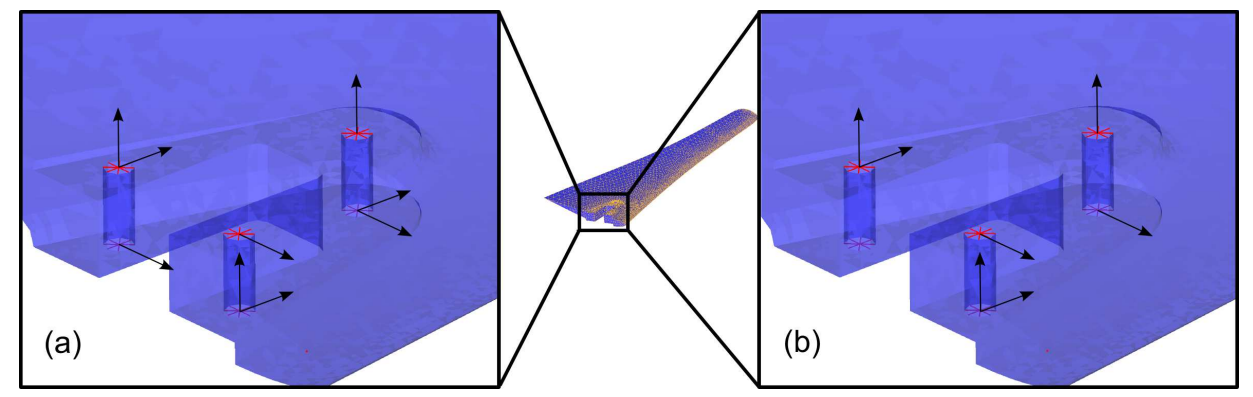

Figure 9: Interface DoFs selected for coupling.

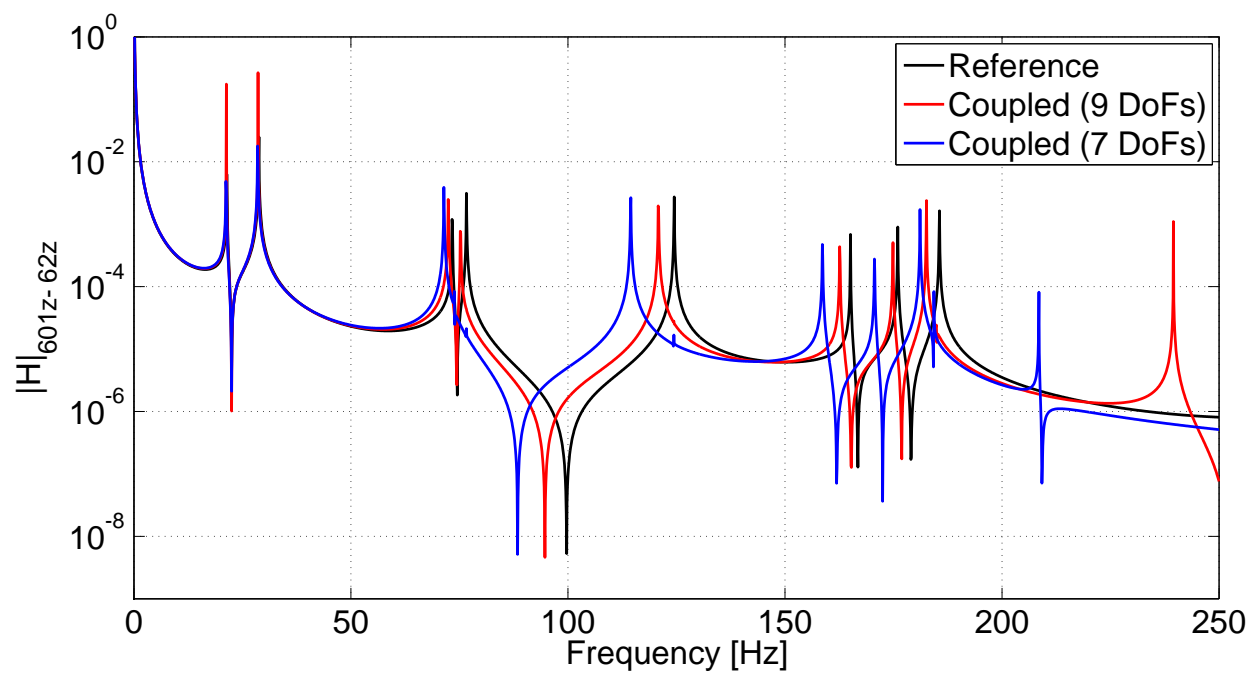

Figure 10: FRF of the coupled system: reference (-), after coupling 1 blade at 9 DoFs (-) or 7 DoFs (一).

\subsection{Coupling the three blades with the hub}

The study carried out on a single blade is extended to the problem of coupling the three blades with the hub. Now, three coupling interfaces have to be considered. To manage all coupling interfaces in a similar manner, a different reference system is introduced for each coupling interface, having the $y$ axis parallel to the longitudinal direction of the blade, the $z$ axis perpendicular to the coupling surface, and the $x$ axis defined accordingly. Therefore, the receptance matrix considered for any of the three blades is always the same, whereas the receptance matrix of the

TABLE 2: Natural frequencies of the coupled system (1 blade with the rest of the rotor).

\begin{tabular}{|c|c|c|c|c|c|}
\hline \multirow{2}{*}{$\begin{array}{l}\text { Mode } \\
\text { number }\end{array}$} & \multirow{2}{*}{$\begin{array}{l}\text { Reference } \\
{[\mathrm{Hz}]}\end{array}$} & \multicolumn{2}{|c|}{7 DoF } & \multicolumn{2}{|c|}{9 DoF } \\
\hline & & {$[\mathrm{Hz}]$} & [Err\%] & {$[\mathrm{Hz}]$} & [Err\%] \\
\hline 7 & 21,4 & 21,2 & $-0,93 \%$ & 21,3 & $-0,47 \%$ \\
\hline $8-9$ & 28,8 & 28,4 & $-1,39 \%$ & 28,6 & $-0,69 \%$ \\
\hline 10 & 73,3 & 71,4 & $-2,59 \%$ & 72,4 & $-1,23 \%$ \\
\hline $11-12$ & 76,6 & 73,8 & $-3,66 \%$ & 75,2 & $-1,83 \%$ \\
\hline $13-14$ & 124,5 & 114,5 & $-8,03 \%$ & 120,8 & $-2,97 \%$ \\
\hline 15 & 165,1 & 158,6 & $-3,94 \%$ & 162,6 & $-1,51 \%$ \\
\hline 16 & 176 & 170,6 & $-3,07 \%$ & 174,9 & $-0,62 \%$ \\
\hline $17-18$ & 185,6 & 181,1 & $-2,42 \%$ & 182,6 & $-1,62 \%$ \\
\hline
\end{tabular}




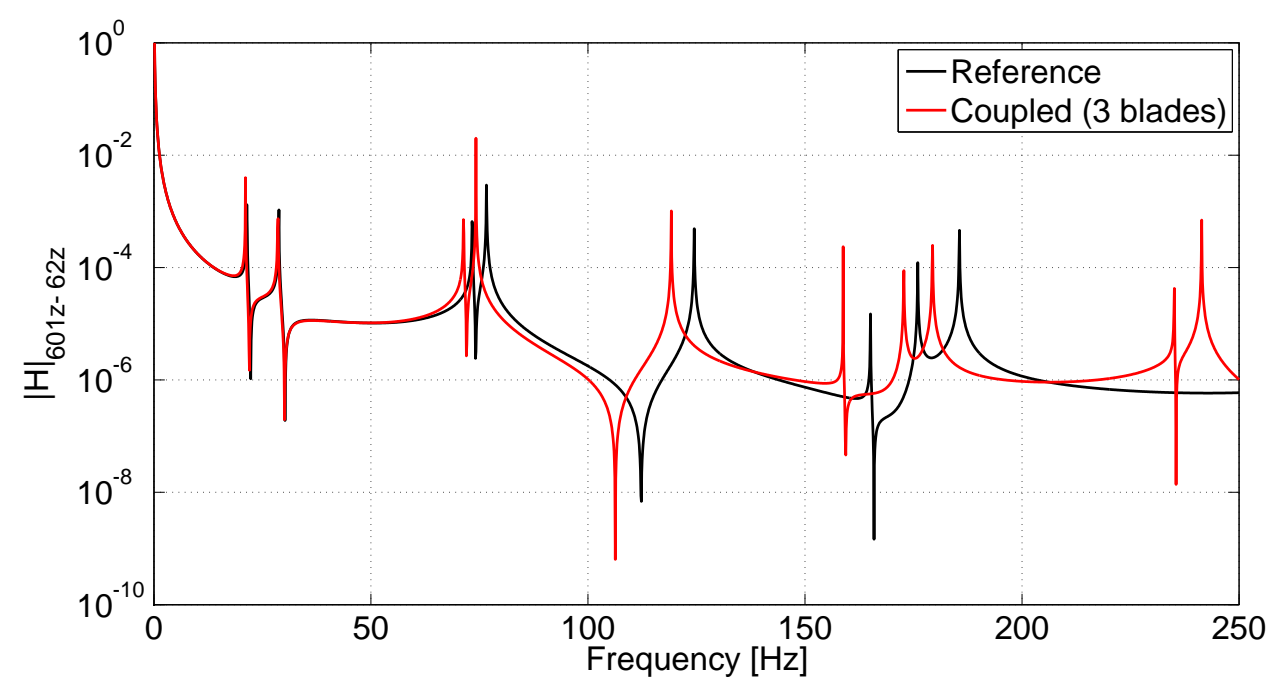

Figure 11: FRF of the coupled system: reference (-), after coupling 3 blades (-).

hub, rotated in these references systems, highlights the axial symmetry of the hub.

$$
H^{H}=\left[\begin{array}{ccc}
H^{\prime} & H^{\prime \prime} & H^{\prime \prime \prime} \\
H^{\prime \prime \prime} & H^{\prime} & H^{\prime \prime} \\
H^{\prime \prime} & H^{\prime \prime \prime} & H^{\prime}
\end{array}\right]
$$

The receptance matrices of the hub and the blades have the the following sizes: $\mathbf{H}^{\mathbf{H}}=[54 \times 54]$ and $\mathbf{H}^{\mathbf{B}}=[54 \times 54]$. In the formulation of the coupling problem by considering all the coupling DoFs, the matrices $\mathbf{H}$ and $\mathbf{B}$ are as follows:

$$
\begin{aligned}
& \mathbf{H}=\left[\begin{array}{llll}
\mathbf{H}_{[54 \times 54]}^{\mathbf{H}} & 0_{[54 \times 54]} & 0_{[54 \times 54]} & 0_{[54 \times 54]} \\
0_{[54 \times 54]} & \mathbf{H}_{[54 \times 54]}^{\mathbf{B}} & 0_{[54 \times 54]} & 0_{[54 \times 54]} \\
0_{[54 \times 54]} & 0_{[54 \times 54]} & \mathbf{H}_{[54 \times 54]}^{\mathbf{B}} & 0_{[54 \times 54]} \\
0_{[54 \times 54]} & 0_{[54 \times 54]} & 0_{[54 \times 54]} & \mathbf{H}_{[54 \times 54]}^{\mathbf{B}}
\end{array}\right] \\
& \mathbf{B}=\left[\begin{array}{lllllllll}
\mathbf{I}_{[18 \times 18]} & 0_{[18 \times 18]} & 0_{[18 \times 18]} & -\mathbf{I}_{[18 \times 18]} & 0_{[18 \times 36]} & 0_{[18 \times 18]} & 0_{[18 \times 36]} & 0_{[18 \times 18]} & 0_{[18 \times 36]} \\
0_{[18 \times 18]} & \mathbf{I}_{[18 \times 18]} & 0_{[18 \times 18]} & 0_{[18 \times 18]} & 0_{[18 \times 36]} & -\mathbf{I}_{[18 \times 18]} & 0_{[18 \times 36]} & 0_{[18 \times 18]} & 0_{[18 \times 36]} \\
0_{[18 \times 18]} & 0_{[18 \times 18]} & \mathbf{I}_{[18 \times 18]} & 0_{[18 \times 18]} & 0_{[18 \times 36]} & 0_{[18 \times 18]} & 0_{[18 \times 36]} & -\mathbf{I}_{[18 \times 18]} & 0_{[18 \times 36]}
\end{array}\right]
\end{aligned}
$$

The FRF of the whole system coupled by means of the 9 DoFs selected above is shown in Fig. 11 compared to the reference FRF of the rotor. Moreover, natural frequencies of the coupled system are shown in Table 3, compared to the reference ones.

\subsection{Decoupling}

In this case, the dynamic behavior of the 3 blades is found starting from the dynamic behavior of the whole rotor (Fig. 5) and of the central hub (Fig. 3). The problem is tackled in the simplest way, by using the standard interface: 
TABLE 3: Natural frequencies of the 3 blades coupled system [Hz]

\begin{tabular}{ll|lc}
\hline $\begin{array}{l}\text { Mode } \\
\text { number }\end{array}$ & $\begin{array}{l}\text { Reference } \\
{[\mathrm{Hz}]}\end{array}$ & \multicolumn{2}{|c}{ Coupl. 3 Blades } \\
{$[\mathrm{Hz}]$} & {$[$ [Err\%] } \\
\hline 7 & 21,4 & 21,1 & $-1,40 \%$ \\
$8-9$ & 28,8 & 28,6 & $-0,69 \%$ \\
10 & 73,3 & 71,3 & $-2,73 \%$ \\
$11-12$ & 76,6 & 74,2 & $-3,13 \%$ \\
$13-14$ & 124,5 & 119,2 & $-4,26 \%$ \\
15 & 165,1 & 158,8 & $-3,82 \%$ \\
16 & 176 & 172,8 & $-1,82 \%$ \\
$17-18$ & 185,6 & 179,4 & $-3,34 \%$ \\
\hline
\end{tabular}

only the DoFs that physically connect the residual substructure (the hub) and the unknown subtructure (the 3 blades) are used as interface DoFs. In this way, all decoupling techniques bring to the same results. To decouple the systems in order to find the dynamic behavior of the blades, the dual decoupling technique is used. It is expressed by the following relation that allows to find the receptance matrix of the unknown system:

$$
\mathbf{H}^{3 \mathbf{B}}=\left[\begin{array}{cc}
\mathbf{H}^{\mathbf{R}} & 0 \\
0 & -\mathbf{H}^{\mathbf{H}}
\end{array}\right]-\left[\begin{array}{cc}
\mathbf{H}^{\mathbf{R}} & 0 \\
0 & -\mathbf{H}^{\mathbf{H}}
\end{array}\right]\left[\begin{array}{l}
\mathbf{B}^{\mathbf{R}^{T}} \\
\mathbf{B}^{\mathbf{H}^{T}}
\end{array}\right]\left(\left[\begin{array}{ll}
\mathbf{B}^{\mathbf{R}} & \mathbf{B}^{\mathbf{H}}
\end{array}\right]\left[\begin{array}{cc}
\mathbf{H}^{\mathbf{R}} & 0 \\
0 & -\mathbf{H}^{\mathbf{H}}
\end{array}\right]\left[\begin{array}{l}
\mathbf{B}^{\mathbf{R}^{T}} \\
\mathbf{B}^{\mathbf{H}^{T}}
\end{array}\right]\right)^{+}\left[\begin{array}{cc}
\mathbf{B}^{\mathbf{R}} & \mathbf{B}^{\mathbf{H}}
\end{array}\right]\left[\begin{array}{cc}
\mathbf{H}^{\mathbf{R}} & 0 \\
0 & -\mathbf{H}^{\mathbf{H}}
\end{array}\right]
$$

where $\mathbf{H}^{\mathbf{R}}$ is the receptance matrix of the whole rotor and $\mathbf{H}^{\mathbf{H}}$ is the receptance matrix of the hub. The previous matrices are both defined only for the standard interface DoFs, which connect the residual substructure and the unknown substructure to each other and their size is [54 $\times 54$ ]. In this analysis, 60 modes for the whole rotor and 16 modes for the hub are taken into account. Furthermore:

$$
\left[\begin{array}{ll}
\mathbf{B}^{\mathbf{R}} & \mathbf{B}^{\mathbf{H}}
\end{array}\right]=\left[\begin{array}{ll}
\mathbf{B}^{\mathbf{R}} & \mathbf{B}^{\mathbf{H}}
\end{array}\right]=\left[\begin{array}{cccccc}
\mathbf{I}_{[18 \times 18]} & 0_{[18 \times 18]} & 0_{[18 \times 18]} & -\mathbf{I}_{[18 \times 18]} & 0_{[18 \times 18]} & 0_{[18 \times 18]} \\
0_{[18 \times 18]} & \mathbf{I}_{[18 \times 18]} & 0_{[18 \times 18]} & 0_{[18 \times 18]} & -\mathbf{I}_{[18 \times 18]} & 0_{[18 \times 18]} \\
0_{[18 \times 18]} & 0_{[18 \times 18]} & \mathbf{I}_{[18 \times 18]} & 0_{[18 \times 18]} & 0_{[18 \times 18]} & -\mathbf{I}_{[18 \times 18]}
\end{array}\right]
$$

Also in this case, three different reference systems - one for each blade - are introduced to express the FRFs in coupling nodes. The three blades that represent the unknown structure are not connected together, so that the receptance matrix of the unknown system $\mathbf{H}^{3 \mathrm{~B}}$ is expected to be a block diagonal matrix composed by three matrices nominally identical to each other, because of the radial symmetry:

$$
\mathbf{H}^{3 \mathbf{B}}=\left[\begin{array}{ccc}
\mathbf{H}^{\mathrm{B} 1} & \approx 0 & \approx 0 \\
\approx 0 & \mathbf{H}^{\mathbf{B} 2} & \approx 0 \\
\approx 0 & \approx 0 & \mathbf{H}^{\mathrm{B} 3}
\end{array}\right]
$$

The decoupling problem is solved using all the translational DoFs of the reduced interface. The identified dynamic behavior (Fig. 12) of the blade reproduces exactly the reference behavior of the blade except for frequencies between 0 and $13 \mathrm{~Hz}$. This is probably due to numerical problems related to the computation of rigid body modes, that are often troublesome for FE software. The dashed line represents the FRF between two DoFs belonging to different blades. As stated previously, it should be theoretically zero. Actually, due to the approximations involved in the use of the reduced interface, it is not zero, but it is several orders of magnitude lower than other FRFs, except at resonances, whose amplitude is not significant being the model undamped. 


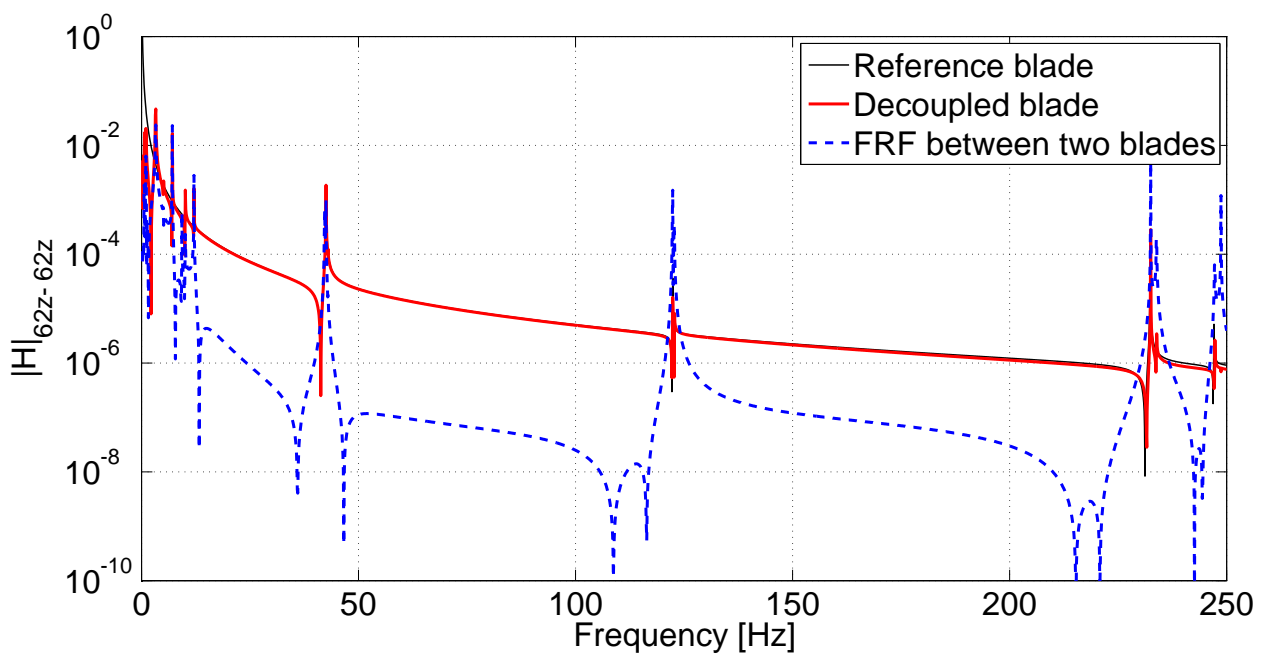

Figure 12: FRF of a blade: reference (-), after decoupling (-); FRF between two different blades (- - -).

\section{SUMMARY AND DISCUSSION}

In this paper, the rotor of the Ampair 600 wind turbine is considered as a test bed for dynamic substructuring, with the aim of selecting a reduced set of relevant DoFs to describe the interface between each blade and the hub. The connection between these two items is ensured by mechanical contact, and therefore compatibility should be enforced between all facing nodes on the contact surface. A first significant reduction is achieved by enforcing compatibility only at the translational DoFs of 6 points, representing the edges of the holes trough which the blade is bolted to the hub. Such simplified connection (18 DoFs for each blade) introduces a relatively low error in the natural frequencies in the range $0-250 \mathrm{~Hz}$. A second reduction is achieved by enforcing compatibility only at 9 DoFs, selected so as to obtain a behavior similar to that of a rigid joint: the additional error introduced by this operation is quite low. Any further attempt to reduce the number of interface DoFs drastically increases the error.

The reduced 9 DoFs interface provides good results in both the considered coupling cases: (a) hub plus two blades coupled with one blade and (b) hub coupled with three blades. In the decoupling case, good results are obtained using the 18 DoFs interface, meaning that the implementation of the procedure is correct. The next step will consider the use of the reduced 9 DoFs interface.

Further developments are envisaged in several directions. First of all, the model of the blade is not very precise, since it considers a homogeneous and isotropic fiber reinforced polyester. On the contrary, it has been pointed out that the reinforcing glass fabric is placed only in the outer part of the blade, and therefore a non homogeneous orthotropic material should be considered. A second line of development is to consider noise polluted FRFs to check the robustness of the technique. Thirdly, experimentally measured FRFs should be used for sub structuring. In fact, the use of computational FRFs is justified by the need of checking the effect of each simplifying assumption, but in view of the use of experimental FRFs.

\section{ACKNOWLEDGEMENTS}

This research is supported by grants from University of Rome La Sapienza and University of L'Aquila. 


\section{REFERENCES}

[1] D'Ambrogio, W. and Sestieri, A., A Unified Approach to Substructuring and Structural Modification Problems, Shock and Vibration, Vol. 11, No. 3-4, pp. 295-310, 2004.

[2] Jetmundsen, B., Bielawa, R. and Flannelly, W., Generalised Frequency Domain Substructure Synthesis, Journal of the American Helicopter Society, Vol. 33, No. 1, pp. 55-64, 1988.

[3] de Klerk, D., Rixen, D. J. and Voormeeren, S., General Framework for Dynamic Substructuring: History, Review, and Classification of Techniques, AIAA Journal, Vol. 46, No. 5, pp. 1169-1181, May 2008.

[4] de Klerk, D., Dynamic Response Characterization of Complex Systems through Operational Identification and Dynamic Substructuring, Ph.D. thesis, TU Delft, 2009.

[5] D'Ambrogio, W. and Fregolent, A., Decoupling procedures in the general framework of Frequency Based Substructuring, Proceedings of 27th IMAC, Orlando (U.S.A.), Feb. 2009.

[6] D'Ambrogio, W. and Fregolent, A., The role of interface DoFs in decoupling of substructures based on the dual domain decomposition, Mechanical Systems and Signal Processing, Vol. 24, No. 7, pp. 2035-2048, Oct. 2010, doi:10.1016/j.ymssp.2010.05.007, also in Proceedings of ISMA 2010, 1863-1880, Leuven (Belgium).

[7] Vormeeren, S. N. and Rixen, D. J., A dual approach to substructure decoupling techniques, Proceedings of 28th IMAC, Jacksonville (U.S.A.), Feb. 2010.

[8] Voormeeren, S. N. and Rixen, D. J., A Family of Substructure Decoupling Techniques Based on a Dual Assembly Approach, Mechanical Systems and Signal Processing, Vol. 27, pp. 379-396, Feb 2012, doi: 10.1016/j.ymssp.2011.07.028.

[9] D'Ambrogio, W. and Fregolent, A., Prediction of substructure properties using decoupling procedures, Structural Dynamics - Eurodyn 2005, Proceedings of the 6th International Conference on Structural Dynamics, edited by Soize, C. and Schuëller, G., pp. 1893-1898, Paris (France), Sep. 2005.

[10] Mayes, R. L. and Arviso, M., Design Studies for the Transmission Simulator Method of Experimental Dynamic Substructuring, Proceedings of ISMA 2010 - International Conference on Noise and Vibration Engineering, edited by Sas, P. and Bergen, B., pp. 1929-1938, Leuven (Belgium), Sep. 2010.

[11] Mayes, R. L. and Ross, M. R., Advancements in hybrid dynamic models combining experimental and finite element substructures, Mechanical Systems and Signal Processing, Vol. 31, pp. 56 - 66, 2012.

[12] D'Ambrogio, W. and Fregolent, A., Promises and pitfalls of decoupling procedures, Proceedings of 26th IMAC, Orlando (U.S.A.), Feb. 2008.

[13] Sjõvall, P. and Abrahamsson, T., Substructure system identification from coupled system test data, Mechanical Systems and Signal Processing, Vol. 22, No. 1, pp. 15-33, 2008.

[14] Mayes, R. L., An Introduction to the SEM Substructures Focus Group Test Bed - The Ampair 600 Wind Turbine, Topics in Experimental Dynamics Substructuring and Wind Turbine Dynamics, Volume 2, edited by Mayes, R., Rixen, D., Griffith, D., De Klerk, D., Chauhan, S., Voormeeren, S., Allen, M. and Proulx, T., Vol. 27 of Conference Proceedings of the Society for Experimental Mechanics Series, pp. 61-70, Springer New York, 2012, 10.1007/978-1-4614-2422-2_7.

[15] Harvie, J. and Avitabile, P., Comparison of Some Wind Turbine Blade Tests in Various Configurations, Topics in Experimental Dynamics Substructuring and Wind Turbine Dynamics, Volume 2, edited by Mayes, R., Rixen, D., Griffith, D., De Klerk, D., Chauhan, S., Voormeeren, S., Allen, M. and Proulx, T., Vol. 27 of Conference Proceedings of the Society for Experimental Mechanics Series, pp. 73-79, Springer New York, 2012, 10.1007/978-1-4614-2422-2_9. 